Asociación de Jóvenes Historiadores y Arqueólogos de Murcia

PANTR REL

REVISTA DE CIENCIA

$$
\begin{array}{r}
Y \\
\text { DIDÁCTICA } \\
\text { DE LA HISTORIA } \\
\text { III - } 2^{2} \text { época }
\end{array}
$$




\section{ASOCIACIÓN DE JÓVENES HISTORIADORES Y ARQUEÓLOGOS DE MURCIA}

\section{PANTA REI. REVISTA DE CIENCIA Y DIDÁCTICA \\ DE LA HISTORIA III. $2^{a}$ época}


CONSEJO EDITORIAL: LAURA ARIAS FERRER

ALEJANDRO EGEA VIVANCOS

ANTONINO GONZÁLEZ BLANCO

RAFAEL GONZÁLEZ FERNÁNDEZ

JOAQUÍN LOMBA MAURANDI

ENRIQUE QUINTANA CIFUENTES

GONZALO MATILLA SÉIQUER

JOSÉ ANTONIO MOLINA GÓMEZ

REDACTOR JEFE:

ÁNGEL LUIS GONZÁLEZ TORRES

Depósito legal: MU-966-1995

I.S.S.N. 1136-2464

Edición de Compobell, S.L. Murcia 


\section{ÍNDICE}

AGRADECIMIENTOS

Presentación

Por Alejandro Egea Vivancos, Laura Arias Ferrer

\section{ARTÍCULOS}

Análisis microscópico de la industria lítica: la traceología

Por Ignacio Martín Lerma

Reflexiones en torno al estudio de la economía en Prehistoria

Por Valentín Martínez García

Aplicaciones SIG en el análisis de las sociedades del pasado. Un caso de estudio: Las primeras comunidades campesinas del Levante Peninsular

Por Gabriel García Atiénzar

La situación actual de los estudios de egiptología en España

Por José Javier Martínez García

La vida cotidiana en el Imperio Asirio. Usos y costumbres de un pueblo que conquistó las Cuatro Regiones del Mundo

Por Ángel Luis González Torres

Las formas de intercambio y las estructuras comerciales orientalizantes en la Vega Baja del Segura: dos variables de estudio arqueológico

Por Sara Pernas García 
Evolución de los patrones de asentamiento en época ibérica. Una propuesta de estudio del mundo ibérico murciano a través del análisis del poblamiento

Hispania Tierra de Roma. Organización y gestión del suelo

Por $M^{a}$ Carmen Santapau Pastor

Hacia una arqueología de la España bizantina. Breves notas a propósito del seminario Work in progress

Por Jaime Vizcaíno Sánchez

Reflexiones acerca de un estudio sobre las estancias auxiliares en la arquitectura barroca catedralicia

Por Francisca del Baño Martínez

La investigación sobre fisiognomía y expresión de las pasiones. Objetivos y metodología

Por María del Mar Albero Muñoz 


\title{
LA SITUACIÓN ACTUAL DE LOS ESTUDIOS DE EGIPTOLOGÍA EN ESPAÑA
}

\author{
José JaVIER MaRTínez GaRCía \\ IPOA-MURCIA
}

\section{Resumen}

La egiptología en España ha sufrido un gran retraso a nivel de investigación y divulgación en su historia por diferentes razones. A día de hoy esta disciplina en España necesita una revisión desde la base, es decir, partiendo de la visión de conjunto en los diferentes ámbitos y esferas de actuación poder llegar a vislumbrar cuales son las mejores soluciones para una potenciación de esta disciplina.

Palabras clave: Egipto, Egiptología, Universidad, Instituto, Asociación.

\begin{abstract}
Egyptology in Spain has suffered a long delay at the level of research and outreach in its history for various reasons. Today this discipline in Spain need a review from the base, on the overview of the different areas and spheres of activity able to see what the best solutions for a strengthening of this discipline.
\end{abstract}

Key words: Egypt, Egyptology, University, Institute Association.

\section{Introducción}

La egiptología, como disciplina que estudia la cultura y el conocimiento del Egipto Antiguo, tiene ya suficiente continuidad en el tiempo como para que en España 
hubiera cierta iniciativa impulsora y de amparo institucional, por el contrario, en este sentido, no sólo no lo ha desarrollado sino que tampoco lo ha hecho con otras como la arqueología produciéndose un retraso escandaloso en nuestro país.

No pretendemos revisar aquí la historia de la egiptología en España, más bien remarcar cual es la situación actual de la misma a través de sus instituciones, asociaciones y personalidades más importantes, ya que son estos quienes realizan una labor fundamental de investigación y divulgación de la egiptología a pesar de encontrarse en una situación de desventaja respecto de otros países europeos vecinos con mas tradición en la disciplina. Partiendo de esta visión de conjunto el lector podrá llagar a sus propias conclusiones respecto a la situación actual de la egiptología en España.

\section{Historia breve de la egiptología en España}

El origen de la egiptología en España lo encontramos en las descripciones de viajeros españoles: así cabe mencionar a Domingo Badía (1767) por su obra «Viajes del Principe Ali Bey El Abbasí en Marruecos, Trípoli, Chipre, Egipto, Arabia Siria y Turquía, escritos por él mismo e ilustrados con mapas y numerosos grabados» Valencia, 1836.

No será hasta 1869 cuando Juan de Dios de la Rada y Delgado adquiera diversas piezas para el Museo Arqueológico Nacional. Tras esto surgirá el «Compendio de Historia Universal - Edad prehistórica y Periodo Orienta» de D. Manuel Sales y Ferré, Catedrático de Sevilla, siguiéndole también en esta línea Miguel Morata con «Discurso de la solemne Inauguración del Curso académico de 1884 a 1885» dedicado a Egipto, posteriormente vendrá José Ramón Mélida y su conferencia « La Religión Egipcia».

En una situación destacada se encuentra Eduardo Toda y Güell, quien realizará una actividad arqueológica en Egipto, de 1884 a 1886, siendo así el primer arqueólogo egiptólogo español, aportando posteriormente sus publicaciones como «A través de Egipto» y «Estudios Egiptológicos».

En 1890 cabe destacar los estudios del Padre Félix Rougier en su obra «Biblia y Egiptología», así como el trabajo de Francisco Gómez del Campillo «Consideraciones acerca del Derecho Egipcio».

También a nivel filológico encontramos los estudios de Manuel Treviño de 1909 «La Escritura Egipcia y su Transcripción Castellana en Caracteres Neo-latinos».

Posteriormente se publicaran otros estudios como «La química y la Farmacia entre los egipcios» por Antonio Murúa en 1910, así como la «Historia de El Arte Egipcio» de Mélida o «La Historia, Teoría y Técnica Ornamental y decorativa en Egipto» de Ricardo Agrasot en 1909. 
Después podemos encontrar estudios concretos como el de Blanco y Caro sobre la escultura de Nectanebo I.

Destaca el Padre Celada quien por su formación en Egiptología dio clases de Historia y arqueología del próximo oriente en la universidad complutense y después en el CSIC y quien fundo el Instituto de Estudios orientales y africanos. Por último, destacar a Eduardo Alfonso Hernán quien publicó una gramática Jeroglífica egipcia «Compendio de Gramática Jeroglífica del Egipcio clásico».

A partir de 1960 se crea un comité español para el salvamento de los tesoros de Nubia dirigido por Martín almagro Basch, realizándose por entonces la excavación de la fortaleza de Sheik Daud, después la necrópolis de Masmás y posteriormente por parte de Presedo Velo y M Pellicer una necrópolis en Nubia. A estos le siguió Rafael Blanco Caro así como otras excavaciones por Presedo en la Nubia sudanesa.

Por otra parte el gobierno colaboró económicamente en el salvamento de Abu Simbel y el gobierno egipcio en agradecimiento le donó el Templo de Dedod en 1970. Cuatro años antes Almagro Basch consiguió permiso para excavar en Hereclaópolis Magna, participando en sucesivos años Jesús López, Presedo Velo, Fernando Fernández y finalmente en 1984 se hace cargo la actual directora Maria del Carmen Pérez Die.

A partir de 1992 se autoriza a Josep Padró a excavar en Oxyrrinchos junto con Mahmud Hamza dentro de una colaboración arqueológica, así en 1994 Luis García Moreno entra como codirector en la excavación de Tell Ibrahim Awad.

\section{Situación Actual de la egiptología en España}

Partiendo de estas primeras investigaciones y actuaciones arqueológicas en Egipto procederemos a concretar las líneas de trabajo de las diferentes instituciones o agrupaciones españolas.

\section{Instituciones oficiales}

Mencionaremos aquí únicamente los estudios de egiptología desde carreras como Licenciatura de Historia, Humanidades o similares, sin mencionar otras licenciaturas, ya que también es posible estudiar egiptología desde el punto de vista de la Historia del Arte.

\section{Las Universidades $^{1}$}

De un total de 74 universidades entre públicas, privadas y de la Iglesia dentro del territorio español, estas son las que mínimamente contemplan en su plan de estudios

1 Aquellas Universidades que no aparecen en el listado se debe a que no poseen ningún tipo de asignatura que de alguna manera esté vinculada a la egiptología. Tampoco hemos incluido en los listados aquellas asignaturas referidas a arqueología a excepción de los casos concretos. 
un pequeño grupo de asignaturas relacionadas. Remarcaremos aquí las asignaturas vinculadas a la egiptología de manera directa o parcialmente directa dentro de dicha asignatura. Por orden de asignaturas e importancia estimada en formación:

\begin{tabular}{|c|c|}
\hline $\begin{array}{l}\text { Universidad de Barcelona } \\
\text {-Arqueología del Próximo Oriente } \\
\text { Antiguo. } \\
\text {-Historia i Civilización egipcias. Historia } \\
\text { de Egipto baja época. } \\
\text {-Lengua I escritura egipcia I. } \\
\text {-Lengua II escritura egipcia II. } \\
\text {-Historia y civilización egipcias. De los } \\
\text { Orígenes al Imperio antiguo. } \\
\text {-Historia y civilización egipcias. Del } \\
\text { Imperio antiguo al imperio medio. }\end{array}$ & $\begin{array}{l}\text { Universidad de Murcia } \\
\text {-Escrituras antiguas y testimonio Histórico. } \\
\text {-Historia del Próximo Oriente Antiguo. } \\
\text {-Arqueología del Antiguo Egipto. } \\
\text {-Fuente del Próximo Oriente Antiguo. } \\
\text {-Antropología del Próximo Oriente Antiguo. }\end{array}$ \\
\hline $\begin{array}{l}\text { Universidad Autónoma de Madrid } \\
\text {-Egiptología. Introducción y Fundamentos } \\
\text {-Arqueología I (Medio Oriente y Egipto) }\end{array}$ & $\begin{array}{l}\text { Universidad de Alicante } \\
\text {-Arqueología del Próximo Oriente Antiguo. } \\
\text {-Historia Antigua del Próximo Oriente. } \\
\text {-Historia de Egipto. }\end{array}$ \\
\hline $\begin{array}{l}\text { Universidad Complutense de Madrid } \\
\text {-Historia de Egipto }\end{array}$ & $\begin{array}{l}\text { Universidad de la Laguna } \\
\text {-Egiptología }\end{array}$ \\
\hline $\begin{array}{l}\text { Universidad de Valladolid } \\
\text {-Antiguo oriente asiático y Egipto }\end{array}$ & $\begin{array}{l}\text { Universidad de Alcalá } \\
\text {-Egiptología }\end{array}$ \\
\hline $\begin{array}{l}\text { Universidad de León } \\
\text {-Historia Antigua del próximo oriente y } \\
\text { Egipto }\end{array}$ & $\begin{array}{l}\text { Universidad de Cantabria } \\
\text {-Historia y civilización de Egipto }\end{array}$ \\
\hline $\begin{array}{l}\text { Universidad de Zaragoza } \\
\text {-Historia del próximo oriente antiguo }\end{array}$ & $\begin{array}{l}\text { Universidad de Sevilla } \\
\text {-Historia de Antiguo Egipto }\end{array}$ \\
\hline $\begin{array}{l}\text { Universidad de Málaga } \\
\text {-Arqueología del próximo oriente y Egipto }\end{array}$ & $\begin{array}{l}\text { Universidad de Huelva } \\
\text {-Historia e Instituciones del Próximo oriente } \\
\text { Antiguo }\end{array}$ \\
\hline $\begin{array}{l}\text { Universidad de Granada } \\
\text {-Historia de Egipto y del próximo oriente } \\
\text { antiguo }\end{array}$ & \\
\hline
\end{tabular}


En la tabla anterior se puede observar las pocas opciones que para estudiar egiptologia se ofrece en las Universidades españolas, dificultando la posibilidad de formar a futuros egiptólogos, al menos considerando una formación arqueológica e histórica.

Por otro lado algunas de estas universidades o instituciones vinculadas a Universidades ofrecen postgrados de especialización en egiptología, como:

La Universidad Autónoma de Barcelona: Master Propio en Egiptología.

Instituto virtual de ciencias Humanas: Master en Egiptología.

Fundación Clos: Master en lengua, historia y cultura del antiguo Egipto.

\section{CSIC: Consejo Superior de Investigaciones Científicas}

Dentro del CSIC tenemos el Instituto de Lenguas y Culturas del Mediterráneo y Oriente Próximo con el proyecto Djehuty, en Luxor, dirigido por José Manuel Galán².

\section{$\underline{\text { Museos y colecciones }}$}

Los principales museos y colecciones españolas son:

- Museo Arqueológico Nacional: Contiene una colección de aproximadamente 1500 objetos de diversas cronologías y materias.

- Museo del Oriente Bíblico del Monasterio de Monserrat

- Museo Egipci (Clos) de Barcelona.

Tras estos podemos encontrar en casi todos los museos provinciales y privados pequeñas colecciones de cerámicas, amuletos, también alguna pieza mayor significativa, pero en general pequeñas piezas, que quizás en un futuro y partiendo del proyecto Domus ${ }^{3}$ podamos tener conjuntamente en una sola base de datos nacional.

Algunos de estos museos con colecciones son:

Museo del Prado.

Museo Balaguer.

Real Academia de historia de Madrid.

Real Academia de bellas artes de San Fernando.

Museo Diocesano de Vich.

2 Proyecto a aclarar en el subapartado de excavaciones españolas.

3 Sistema de documentación de Museos informatizado. 
Museo Arqueológico de Huesca.

Museo Arqueológico de La Coruña.

Museo de la Academia de Historia de Córdoba.

Museo de Málaga.

Museo Arqueológico de granada.

Museo Arqueológico de Murcia.

Museo de Bellas Artes de Castelló de la Plana.

Museo Municipal de Almassora.

Museo de l'Art de la Pell de Vic.

Museo de les Artes Decorativas de Barcelona.

Museo Cau Ferrat de Sitges.

Museo Textil de Terrassa en Barcelona.

Museo de Montserrat en Barcelona.

Museo del Perfume de Barcelona.

Museo Arqueológico de Catalunya.

Museo Municipal Can Xifreda de Sant Feliu de Codines.

Museo Episcopal de Vic.

Biblioteca Museo Victor Balaguer de Vilanova y la Geltrú.

Museo Comarcal del Maresme de Mataró.

Empuries. Museu d'Arqueologia de Catalunya.

Girona. Museu d'Arqueologia de Catalunya.

Peralada. Museu del Castell, Museu del Vidra.

Ullastret - Museo d'Arqueologia de Catalunya.

Institut d'Estudis Ilerdencs en Lleida.

Museo Comarcal del Baix Ebre i del Montsia en Amposta.

Museu Bíblic Tarraconense.

Museu Nacional Arqueològic de Tarragona.

Museo Arqueològic del Vendrell.

Museo Bíblico de Palma de Mallorca.

Museu d'Arqueologia d'Eivissa i Formentera.

Museo de Menorca.

Institutos de investigación

Instituto del Próximo Oriente Antiguo. IPOA

Es un Instituto Interuniversitario que agrupa a los centro de Barcelona, Murcia y Salamanca, siendo su ámbito de actuación el estudio del Próximo Oriente y Egipto, tanto a nivel divulgativo, docencia y actuación arqueológica. 
Sus sedes respectivas son:

\begin{tabular}{|l|l|}
\hline Institut del Pròxim Orient Antic (IPOA) & Institut del Pròxim Orient Antic \\
Universitat de Barcelona & Universitat Autònoma de Barcelona \\
Gran Via de les Corts Catalanes 585 & Edifici B - Campus de la UAB \\
08007 Barcelona (Spain) & 08193 Bellaterra (Barcelona) (Spain) \\
Tel./Fax (+34) 93 4035697 & Tel. (+34) 93 5812384 \\
ipoa@ub.edu & Fax: (+34) 93 5813114 \\
http://www.ub.edu/ipoa & http://www.uab.es/iepoa/ \\
\hline Instituto del Próximo Oriente Antiguo & Instituto del Próximo Oriente \\
Universidad de Murcia & Antiguo \\
Antiguo Edificio Escuela de & Universidad de Salamanca \\
Empresariales & Facultad de Filología \\
Actor Isidoro Máiquez, 8 - Vistalegre & Departamento de Filología Clásica e \\
30007 Murcia (Spain) & Indoeuropeo \\
Tel./Fax: (+34) 968 363890 & Plaza de Anaya, s/n \\
ipoa@um.es & 37001 Salamanca (Spain) \\
http://www.um.es/ipoa/ & T. (923) 29 44 45 (ext. 1701) \\
& Fax: (923) 29 45 09 \\
\hline
\end{tabular}

\section{Instituto Egipcio de Estudios Islámicos}

El Instituto Egipcio de Estudios Islámicos, Mediterráneos e Iberoamericanos, fue fundado por el Gobierno Egipcio en el año 1.950 en Madrid, para trabajar a nivel académico y cultural en los campos de la cultura árabe e hispana y mediterránea a través de Boletín de Prensa Cultural, Publicaciones, Revista del Instituto Egipcio de Estudios Islámicos, Biblioteca, Jornadas, Cursos y Conferencias, Exposiciones de Arte y Cursos de Lengua Árabe.

Actualmente su sede se encuentra en:

Francisco de Asís Méndez Casariego, 1 - 28002 Madrid

Tlf: 915639468 - 915636782 - Fax: 915638640

e-mail: iegipcio@mundivia.es

\section{Instituto Catalán de Arqueología Clásica ICAC}

Centro de investigación de la arqueología clásica creado por la Generalitat de Cataluña y la Universidad Rovira y Virgili, con la participación del Consejo Interu- 
niversitario de Cataluña. Siendo su finalidad la investigación, formación y difusión de la civilización y culturas clásicas. En base a esta idea fundamental colabora en la excavación de la Misión de Oxirrinco.

Actualmente su sede se encuentra en:

Plaça d'en Rovellat, s/n 43003 - Tarragona

Tel. 977249133 Fax 977224401

info@icac.net

\section{Instituto Virtual de Ciencias humanas}

Instituto con la intención de llenar la laguna de formación en materias interdisciplinares relacionadas con las llamadas ciencias humanas.

Dentro de su formación ofrece cursos de egiptología y master.

http://www.ivch-cursos.com

Entre sus profesores de master cuentan con:

José Ramón Pérez-Accino

José Félix Alonso García

Carlos González Wagner

Ana Isabel Navajas Jiménez

Antonio Pérez Largacha

Juan Cortés Martín

Jesús J. Urruela Quesada

\section{Instituciones privadas}

A continuación presentamos una serie de instituciones privadas, algunas de ellas muy consagradas a nivel nacional, como la Asociación española de Egiptología o la Societat Catalana d Egiptología.

\section{Asociación española de Egiptología.}

La Asociación Española de Egiptología es una institución científica dedicada a la formación egiptológica, fundada el 29 de Diciembre de 1986. Sus objetivos son: La salvaguarda del Patrimonio Cultural del Egipto Faraónico, la exploración e investigación del material disponible sobre dicha civilización, el fomento de la divulgación y difusión de los conocimientos egiptológicos dentro del ámbito territorial del Estado Español, la defensa y promoción de la Cultura Faraónica y la colaboración con Instituciones Nacionales e Internacionales dedicadas a la actividad egiptológica. 
Su sede se encuentra en:

Paseo de la Habana, $174^{\circ} \mathrm{D}$

28036 - Madrid

Tlf: 915616320

\section{$\underline{\text { Societat catalana de egiptología }}$}

La Societat Catalana de Egiptología es una asociación cultural, dedicada al estudio y divulgación del Antiguo Egipto, que ofrece actividades como cursos, conferencias, seminarios y cursillos a cargo de profesores universitarios o personalidades relacionadas con la egiptología, organiza viajes a Egipto y a otros países con interés egiptológico. También publica la revista Nilus (boletín de la SCE) y colabora y participa en las excavaciones arqueológicas de Oxirrinco (Egipto).

$\mathrm{Su}$ sede se encuentra en:

C/ Aragón, 305, entresuelo (08009) Barcelona

Tef: 934578120

\section{Instituto de Estudios Islámicos y del Próximo Oriente}

El Instituto de Estudios Islámicos y del Oriente Próximo centra sus investigaciones en la cultura árabe e islámica y del Oriente Próximo, especialmente en la lengua y literatura árabes, el arte islámico y el antiguo Oriente Próximo.

Su sede se encuentra actualmente en:

c/ Diputados, 19-2150004 Zaragoza, España

Tel.: 976404 722Fax: 976404725

Correo electrónico: info@ieiop.csic.es

\section{Instituto de Estudios del Antiguo Egipto}

Institución científica, sin ánimo de lucro, constituida en Madrid, en Junio de 1997, dedicada a la investigación, preservación, conservación y divulgación del patrimonio del Antiguo Egipto, estando especialmente comprometida con las tareas de conservación del patrimonio arqueológico de las culturas del antiguo Egipto.

En el desarrollo de estas tareas, el I.E.A.E., ha desarrollado, previa invitación de las autoridades competentes, la elaboración de un proyecto de adecuación y presentación de uno de los monumentos más destacables entre las tumbas de particulares ubicadas en la necrópolis de la antigua ciudad de Tebas (Luxor), concretamente de la TT 353, uno de los dos monumentos que el arquitecto Jefe de la reina Hatshepsut (1473-1458 a. de C.) se hizo excavar, convirtiéndose en el proyecto Sen-en-mut. 
Su sede se encuentra en:

C/Goya, $691^{\circ}$ Ext. Izda. (28001) Madrid

Tlf: 915765795

\section{$\underline{\text { Instituto valenciano de Egiptología }}$}

Surge como foco de actividades culturales relacionadas con la Egiptología dentro del ámbito del a Comunidad Valenciana realizando actividades como cursos, conferencias, o edición de la Revista: La Puerta de Maat.

Contacto: info@ivde.org.

\section{Asociación de amigos de la egiptología de Lorca}

Pequeña asociación que celebra cada año las Jornadas de Egiptología de Lorca.

\section{Asociación andaluza de egiptología}

Asociación española cultural sin ánimo e lucro y de ámbito de actuación nacional.

Dentro de sus objetivos esta el estudio, investigación, defensa, difusión y promoción de la cultura del Antiguo Egipto, promocionar y ayudar a la egiptología en España, ayudar a la conservación del Patrimonio cultural e histórico egipcio, así como reunir y distribuir toda la información posible sobre nuevos descubrimientos, expediciones, excavaciones en curso y estudios sobre egiptología.

Su sede se encuentra en:

Calle de la Plaza, $2-1^{\circ} \mathrm{B}$

11130 Chiclana de la Fra. Cádiz.

Instituto Internacional de Egiptología para el Estudio del derecho, tecnología y ciencia en el Antiguo Egipto

Tiene como premisa fundamental la necesidad de combinar ámbitos multidisciplinares en la investigación de muchos de los campos que abarca la Egiptología. Publican la revista electrónica Aegyptus Journal of Egyptology.

$\mathrm{Su}$ sede se encuentra en:

Calle de la Plaza, 2- ${ }^{\circ} \mathrm{B}-11130$

Chiclana de la Fra. Cadiz 


\section{Fundación Clos}

La Fundación Arqueológica Clos nació en el año 1993, por la iniciativa individual de Jordi Clos, a raíz de su pasión por la Egiptología. La Fundación se ha comprometido con la investigación, el estudio y la difusión del antiguo Egipto; y ha desarrollado, con recursos totalmente privados, un amplio abanico de actividades a favor de la cultura y el arte del mundo antiguo. Una de sus mayores aportaciones ha sido la creación del Museo Egipci de Barcelona. Su sede se encuentra en:

FUNDACIÓ ARQUEOLÒGICA CLOS

MUSEU EGIPCI DE BARCELONA

València, 284. E-08007 Barcelona

Tel. (34) $934880188 \cdot$ Fax. (34) 934878060

\section{Las Publicaciones}

A día de hoy existen una serie de publicaciones de mayor o menor nivel científico que contribuyen a la difusión en diferentes niveles de los estudios egiptológicos realizados por españoles en España. Descartaremos aquellas revistar de carácter general dentro de la arqueología que también tienen artículos sobre Egipto, debido a la gran magnitud de las mismas, como la Revista de Arqueología o Historia National Geographic.

Aquí seleccionamos algunas de ellas:

- Aegyptiaca Complutensia: Revista sobre egiptología de la Universidad de Alcalá de Henares

- Aegyptus Journal of Egyptology: Revista del Instituto Internacional de Egiptología para el Estudio del derecho, tecnología y ciencia en el Antiguo Egipto

- Aula Aegyptiaca: Revista publicada por la Universidad Autónoma de Barcelona sobre temas de investigación egiptológicos.

- Aula Orientalis: revista de Estudios del Próximo Oriente Antiguo.

- BAEDE: Boletín de la Asociación española de Egiptología. Madrid

- BAEDO: Boletín de la Asocación Española de Orientalistas.

- BAEO: Boletín de la Asociación española de orientalistas. Madrid.

- BASADE: Boletín anual de la Asociación Andaluza de Egiptología

- BIAE: Boletín Informativo de Amigos de la Egiptología.

- Nilus: Revista de la Societat Catalana de Egiptología.

- Revista Orientalia: revista de divulgación general, que informa de los últimos descubrimientos acerca de las culturas de Asia y norte de África, contemporáneas o del pasado.

- La Puerta de Maat: Revista egiptológica cuatrimestral publicada por el Instituto Valenciano de Egiptología. 
Oxirrinco:

El yacimiento de El Bahnasa se encuentra en la ribera izquierda de Bahr Yussef, a unos $190 \mathrm{Km}$ al sur de El Cairo. Fue descubierto e identificado como la antigua ciudad de Oxirrinco por Denon, uno de los componentes de la expedición de Bonaparte. Desde 1897 se han realizado ocasionalmente excavaciones y ha sido saqueado para la búsqueda de papiros, ya que la gran riqueza de estos documentos ha hecho célebre el yacimiento.

Con motivo del descubrimiento de una tumba de época Saíta en 1982, una Misión arqueológica del Consejo Superior de Antigüedades emprendió las excavaciones en El-Bahnasa, y en 1992 la Misión se convirtió en Mixta por la asociación en los trabajos de la Universidad de Barcelona, dirigiendo los trabajos Josép Padró.

Heracleópolis Magna:

Heracleópolis Magna, Nen-nesut de los antiguos egipcios y actual Ehnasya el Medina, es una ciudad situada en el Egipto Medio, en la provincia de Beni Suef, a $130 \mathrm{kms}$. al sur de El Cairo. En época faraónica fue la capital del XX nomo del Alto Egipto, llamado Naret hentet o Naret superior. La región está atravesada por el Bahr el Yusuf, brazo del Nilo que desemboca en el lago Moeris en el Fayum. Actualmente es dirigida por María del Carmen Pérez Die.

Qubbet el-Hawa:

La necrópolis de Qubbet el-Hawa se halla justo en frente de la moderna ciudad de Asuán, unos mil kilómetros al sur de El Cairo. En ella se han encontrado a lo largo de diferentes campañas de excavación unas 60 tumbas talladas en la roca de la colina, algunas de las cuales no han sido investigadas nunca. Actualmente dirigida por Alejandro Jiménez Serrano.

Djehuty y Hery:

Proyecto que lleva a cabo la excavación, restauración y publicación de las tumbas de Djehuty y Hery en Dra Abu el-Naga, en Luxor. Estas excavaciones se iniciaron en el 2002, realizándose durante un mes en campo y el resto del año en laboratorio. Actualmente las excavaciones están dirigidas por José Manuel Galán

Dyebel Barkal:

Principalmente se llevan a cabo trabajos de restauración al norte del yacimiento y excavaciones arqueológicas en los enterramientos reales y civiles de la necrópolis de la antigua Napata. Los directores actuales son Francesca Berenguer, Monserrat Díaz de Cerio y Luis Manuel Gonzálvez.

Harwa:

Excavación de la tumba de un alto funcionario de Luxor del Reino Antiguo. Excavación dirigida por Miguel Angel Molinero. 


\section{Meidum:}

Trabajos de restauración y conservación de los monumentos de la zona. Concretamente la Mastaba 16. Trabajos dirigidos por Luis Manuel Gonzálvez.

\section{Sen-en-mut:}

Se trata de la TT 353, uno de los dos monumentos que el arquitecto Jefe de la reina Hatshepsut (1473-1458 a. de C.) se hizo excavar en la necrópolis de la ciudad de Tebas en Luxor. Dirigida por Francisco Martín Valentín.

Hay que tener en cuenta que aunque sean pocas las intervenciones propiamente españolas, además existen otras misiones que siendo de titularidad extranjera participan en ellas españoles, de manera que poco a poco la egiptología española se va abriendo camino.

\section{Los egiptólogos}

No nos extenderemos aquí en cada uno de los currícula de los siguientes egiptólogos, únicamente destacar sus proyectos más importantes:

\begin{tabular}{|l|l|}
\hline Josep Padró & $\begin{array}{l}\text { Actualmente dirige las excavaciones de Oxirrinco y es } \\
\text { catedrático de Egiptología en la Universidad de Barcelona. }\end{array}$ \\
\hline Mari Carmen Pérez Die & $\begin{array}{l}\text { Directora de las excavaciones de Heracleópolis Magna y } \\
\text { conservadora del Museo Arqueológico Nacional }\end{array}$ \\
\hline José Manuel Galán & $\begin{array}{l}\text { Científico del CSIC y director de las excavaciones de la tumba } \\
\text { de Djehuty y Hery en Luxor. }\end{array}$ \\
\hline Myriam Seco & $\begin{array}{l}\text { Arqueóloga profesional, trabaja en la excavación del templo } \\
\text { de Amenofis III en Luxor y participa en diversas excavaciones } \\
\text { de carácter de urgencia en Egipto. }\end{array}$ \\
\hline Javier Martínez Babón & Codirector de la tumba 34 de Monthemhat en Tebas oeste. \\
\hline Miguel Ángel Molinero & $\begin{array}{l}\text { Director de la excavación de Harwa y profesor de Historia } \\
\text { Antigua y Egiptología de la Universidad de La Laguna. }\end{array}$ \\
\hline José Ramón Perez-accino & $\begin{array}{l}\text { Profesor de Egiptología de la Universidad College de } \\
\text { Londres }\end{array}$ \\
\hline Alejandro Jiménez Serrano & Director de las excavaciones de Qubbet el-Hawa. \\
\hline Josep Cervelló & Miembro del equipo de Dyebel Barkal. \\
\hline Monserrat Díaz de Cerio & Directora de la excavación de Dyebel Barkal. \\
\hline Esther Pons & $\begin{array}{l}\text { Miembro de la excavación de Oxirrinco y conservadora del } \\
\text { MAN. }\end{array}$ \\
\hline
\end{tabular}




\begin{tabular}{|l|l|}
\hline Elisa Castel & $\begin{array}{l}\text { Miembro de la Internacional Association of Egiptologist, la } \\
\text { Egypt Exploration Society. }\end{array}$ \\
\hline José Miguel Serrano & $\begin{array}{l}\text { Profesor de la Universidad de Sevilla a la vez que participa en } \\
\text { la excavación de Djehuty. }\end{array}$ \\
\hline Maria José López Grande & Miembro del equipo de la Excavación de Djehuty. \\
\hline Francisco Martín Valentín & Director de la excavación de la tumba de Sen-en-mut. \\
\hline Eva Subias & $\begin{array}{l}\text { Miembro del equipo de la excavación de Oxirrinco y profesora } \\
\text { de la Universidad Rovira y Virgili. }\end{array}$ \\
\hline Maite Mascort & $\begin{array}{l}\text { Miembro del equipo de la excavación de Oxirrinco y técnico } \\
\text { arqueóloga del Servei de Arqueología de Catalunya. }\end{array}$ \\
\hline
\end{tabular}

Por supuesto, tras cada uno de ellos existe un equipo multidisciplinar de arqueólogos, filólogos, arquitectos, topógrafos, restauradores, ingenieros, historiadores, historiadores del arte, etc., que contribuyen con su trabajo al avance de la egiptología en España.

\section{Conclusión}

Partiendo de esta aproximación a la situación actual de la egiptología en España podemos deducir varios puntos interesantes:

Primero: En función de la situación actual, tan precaria a nivel de recursos para la investigación y docencia, los investigadores y estudiantes se ven forzados a estudiar y colaborar con otras instituciones extranjeras ya que en estas se tienen mas opciones de trabajo y ejercicio de la profesión.

Segundo: El estado español, desde su ámbito de actuación debería promocionar más las líneas de investigación y docencia en esta línea, creando no ya la Licenciatura en Egiptología que quizás no tendría cabida en la situación actual en relación al plan Bolonia, sino quizás y mas en relación y consonancia con este, la Licenciatura en Arqueología, para que dentro de esta nueva carrera hubiera una especialización en Egiptología, donde se pudieran estudiar asignaturas claves como lengua, arte o arquitectura egipcias, así como aquellas comunes vinculadas a la arqueología y metodología arqueológica que a día de hoy se ve inmersa en una interdisciplinariedad muy compleja.

En esta línea parece que el estado ya ha comenzado a mover ficha al mostrar interés por reabrir el instituto Cervantes de El Cairo, edificio que se encontraba en muy 
malas condiciones y que no cumplía con su cometido y que parece que ahora va a recibir un impulso institucional que permita a este Instituto y sus infraestructuras servir de punto de partida y referencia para la egiptología española en el Cairo, un poco a imagen de otros instituto como el Instituto Francés de Arqueología Oriental (IFAO).

Tercero: Los investigadores actuales tanto privados como de instituciones deberían colaborar mas entre ellos y juntos dedicar su esfuerzo a la divulgación de la cultura egipcia, ya que en la actualidad parece haber una brecha entre los que quieren entrar en los círculos científicos sin haber llevado un camino puramente académico y aquellos que si lo han llevado y reclaman esa parcela para sí, como especialistas, rechazando a los demás.

Cuarto: Por otra parte, hoy día nos encontramos en la sociedad de la información y no resulta tan importante como antes realizar un periplo por las diferentes universidades europeas en busca de cierta documentación de cara a un estudio específico de egiptología, ya que hoy día prácticamente se puede conseguir casi todo por medio de la red y cada día más y más información se puede encontrar en esta; eso no quita que como hábiles investigadores tengamos que «ver la aguja entre la paja» y saber que tomar de la inmensidad de información que tenemos en la red.

Por tanto, deberemos por una parte esforzarnos todos por situar los estudios de egiptología donde se merecen dentro del panorama español mediante un esfuerzo por parte de instituciones y asociaciones interesadas por la cultura y la sociedad del Antiguo Egipto.

\section{Bibliografía}

CASADO RIGALT, D., «José Ramón Mélida y la Egiptología en España (18751825), Boletín de la Asociación Española de Egiptología, no 17, 2007, 23-38.

CLAYTON, P.A., Redescubrimiento del Antiguo Egipto: artistas y viajeros del siglo XIX. Ediciones del Serbal, 1994.

DUEÑAS, J., «A la conquista de Egipto: la arqueología española progresa en tierra de faraones», , Clío: Revista de Historia, nº 72, 2007, 32-46.

GARCÍA I MARRASÉ, N.E., «Fundació arqueològica Clos: aportación de una entidad privada a la egiptología española», Hesperia culturas del Mediterráneo, $\mathrm{n}^{\circ} 4$, 2006, 201-212.

LARA PEINADO, F., «Un cultivador de la Egiptología. José Ramón Mélida», Boletín de la Asociación Española de Egiptología, n 3,1991, 188-193.

LÓPEZ GRANDE, M. J., «El viaje a Egipto: Primeros viajeros españoles y primeras miradas de la investigación española hacia las tierras del Nilo.»Cuadernos de prehistoria y arqueología., no 30, 2004, 225-239. 
LÓPEZ GRANDE, M. J., «Los estudios de Egiptología en España y la Asociación Española de Egiptología». La cristianización del pasado: génesis y desarrollo del marco institucional de la arqueología en España. 1997, 721-728.

MARTIN VALENTÍN, F.J., «La Egiptología Española», Historia 16, 1998, 38-60.

MARTIN VALENTÍN, F.J., «Notas para una historia de la Egiptología en España» Boletín de la Asociación Española de Egiptología, no 4-5, 173-196.

MARTIN VALENTÍN, F.J., «Notas para una historia de la Egiptología en España (II)» Boletín de la Asociación Española de Egiptología, $\mathrm{n}^{\circ}$ 8, 245-258.

MOLINERO POLO, M.A., «Los difíciles comienzos de la egiptología en España: de las colecciones privadas a las primeras expediciones», Clío: Revista de Historia, $n^{\circ} 23,2003,42-49$.

MOLINERO POLO, M.A., «La egiptología en España (y II): los descubrimientos de Nubia y el nacimiento de la egiptomanía», Clío: Revista de Historia, ${ }^{\circ}{ }^{24}, 2003$, 36-43.

TRELLO ESPADA, J., «La Egiptología en España», Boletín de la Asociación Española de Egiptología, no 4-5, 215-220. 